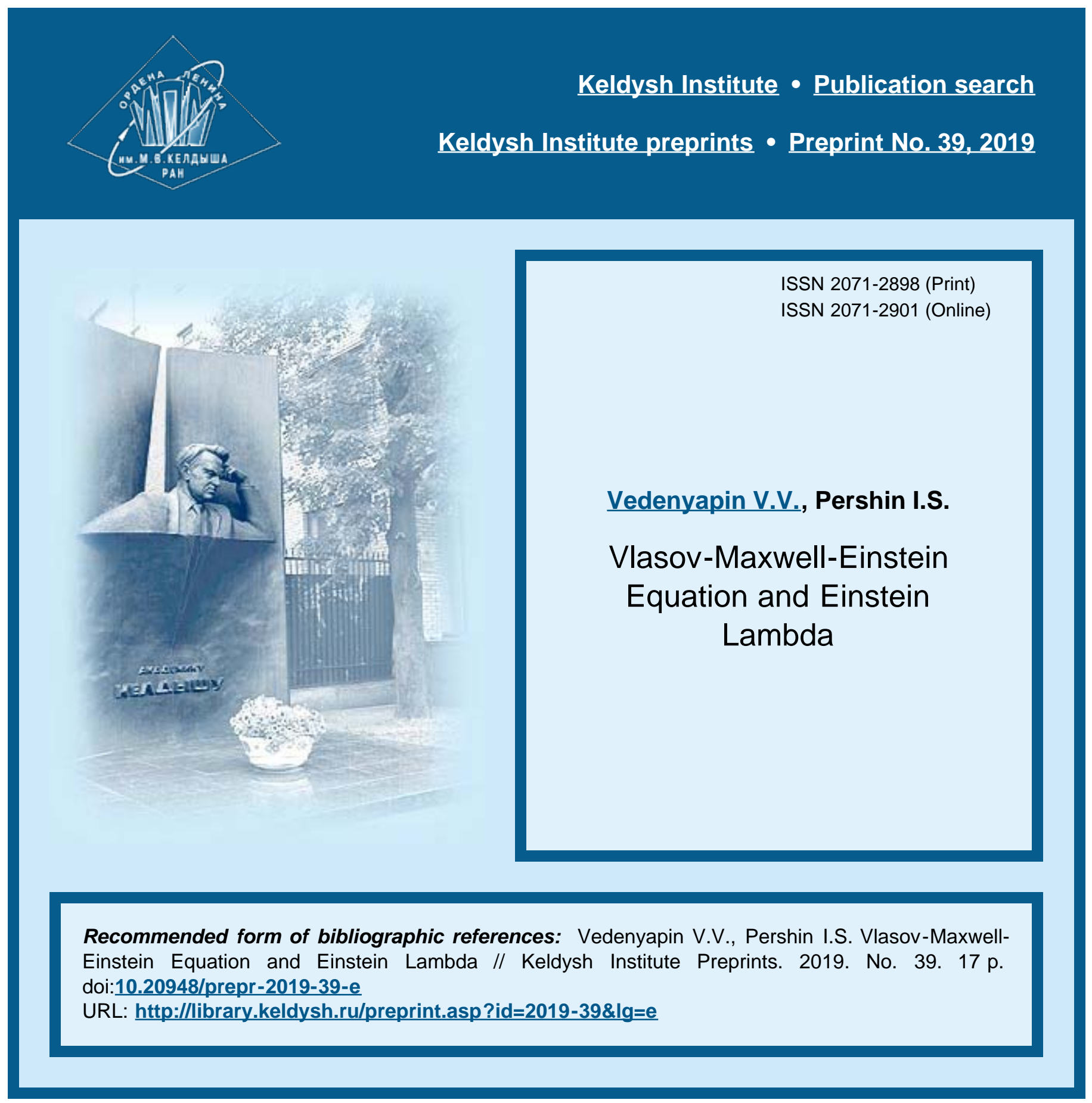




\author{
Ордена Ленина \\ ИНСТИТУТ ПРИКЛАДНОЙ МАТЕМАТИКИ \\ имени М. В. Келдьиша \\ Российской академии наук
}

V.V. Vedenyapin, I.S. Pershin

\title{
Vlasov-Maxwell-Einstein Equation and Einstein Lambda
}

Москва

2019 


\section{УДК 517.958}

Веденяпин В.В., Периин И.С.

\section{Уравнение Власова-Максвелла-Эйнштейна и лямбда Эйнштейна}

Выводятся уравнения Власова-Максвелла-Эйнштейна из классического действия Лоренца-Шваршильда-Гильберта-Эйнштейна. При этом требуется и удаётся синхронизовать собственные времена различных частиц. На основе полученных выражений для действия анализируется лямбда Эйнштейна и вклад её в тёмную энергию.

Ключевые слова: лямбда Эйнштейна, синхронизация времён, тёмная энергия, уравнение Власова, уравнение Власова-Эйнштейна.

\section{Victor Valentinovich Vedenyapin, Ilya Sergeevich Pershin Vlasov-Maxwell-Einstein Equation and Einstein Lambda}

Vlasov-Maxwell-Einstein equations are derived from classical action of LorentzSchwarzschild-Hilbert-Einstein. We need and get synchronization of times of different particles. On the basis of obtained results we analyze Einstein's lambda and its connection with dark energy.

Keywords: Einstein Lambda, synchronization of times, dark energy, Vlasov equation, Vlasov-Einstein equation.

Работа выполнена при финансовой поддержке Министерства науки и высшего образования РФ по программе повышения конкурентоспособности РУДН 5-100 среди ведущих мировых научно-образовательных центров на 2016-2020 гг. и при поддержке программы Президиума РАН № 01 «Фундаментальная математика и ее приложения» (грант PRAS-18-01). 


\section{Introduction}

Einstein Lambda is now widely used to explain dark energy. [1-4, 14, 15, 42, 43]. Einstein himself considered the introduction of the Lambda the biggest mistake of his life. In this paper we show that in the classical action the contribution of other components is difficult to be distinguished from the Lambda's contribution, so Einsteins' suspicions can be justified. To compare these contributions, we need to rewrite some of them through the distribution functions, as is done in the derivation of the Vlasov equations. We derive the Vlasov-Maxwell-Einstein equation [40, 41] via classical actions and Maxwell Lagrangian and Poincaré-Einstein-SchwarzschildHilbert-Pauli Lagrangian.

Vlasov-like equations live an amazing life now. Not only the area of their applications expands all the time, but also new names emerge constantly. There already are the Vlasov-Poisson equations for gravity, plasma and electrons, the Vlasov-Maxwell equations for electrodynamics and the Vlasov-Einstein equations for strongly relativistic gravity. In this paper, we present the Vlasov-Maxwell-Einstein equations. The name choice is natural because it originates from the classical Lagrangians of the general theory of relativity (GTR) and electrodynamics [1-5]. When deriving Vlasov-like equations from the classical Lagrangians [1-5] according to [1-13], the Liouville equations are first derived. In the case of the Vlasov-Maxwell-Einstein equations, new difficulties arise. We need to synchronize times of different particles and compare different forms of Lagrangians for their geodesics. The interval integral appears, which is usually assumed to be unity [1-5]. It is impossible to synchronize the times without this integral, and therefore write down the Vlasov-Einstein equation for many particles. To obtain the self-consistent field equations, it's required to transform the classical actions from Lagrangian coordinates to Eulerian coordinates using distribution functions.

The work plan is as follows. Firstly, we consider the theory of geodesics with an electromagnetic field for classical Lagrangians. In the second section a multiparticle problem leads to time synchronization. We write out the Hamiltonian formulation and write down a Liouville equation. The third part is about the application of the Hamiltonian formalism for time-independent fields. The next section is about the integration of the geodesic equations in fields that depend only on time using the Hamiltonian formalism. In the last section we finally derive the Vlasov-MaxwellEinstein equations. 


\section{The Equivalence of Two Actions in the GTR and in the Theory of Geodesics}

Let's consider the GTR action in the presence of an electromagnetic field [1-5], describing the movement of particles of $m$ mass and $e$ electric charge.

$$
S_{1}=-m c \int \sqrt{g_{a b} \frac{d x^{a}}{d q} \frac{d x^{b}}{d q}}-\frac{e}{c} \int A_{a} \frac{d x^{a}}{d q} d q
$$

Here $g_{a b}(x)$ is a metric in 4-dimensional space-time $x \in R^{4}$, and $A_{a}(x)$ is the potential of an electromagnetic field $(a, b=0,1,2,3)$. Repeating superscripts and subscripts are summed up here and thereafter. Such an action is inconvenient for the Hamiltonian formalism, since its Hamiltonian is zero by the Euler's homogeneous function theorem. Indeed, the Lagrangian is a linear expression in velocities. The transition to a more convenient action is more or less known in the literature [1-4], but wasn't strictly justified, so we will give the proof for it. Take a look at this action

$$
S=-\frac{m c}{2 \sqrt{I}} \int g_{a b}(x) \frac{d x^{a}}{d q} \frac{d x^{b}}{d q} d q-\frac{e}{c} \int A_{a} \frac{d x^{a}}{d q} d q
$$

The value of $I=g_{a b} \frac{d x}{d q} \frac{d x}{d q}$ in constant, as we will see later. The connection of (1) and (2) is justified by the following general lemma.

Consider an action

$$
k \int L\left(x, \frac{d x}{d q}\right) d q+\int L_{1}\left(x, \frac{d x}{d q}\right) d q
$$

and another action

$$
\int h(L)\left(x, \frac{d x}{d q}\right) d q+\int L_{1}\left(x, \frac{d x}{d q}\right) d q,
$$

where $h(L)$ is a some function of $L$ Lagrangian. Let's compare their Euler-Lagrange equations.

The Lemma is as follows: If these conditions are true

1. L Lagrangian is an integral of motion for (3) action.

2. $k$ from (3) must be equal to the derivative of $h(L)$ from (4), that is $k=\frac{d h(L)}{d L}$.

Then (3) and (4) are equivalent, which means that their Euler-Lagrange equations are the same.

The proof is to compare the Euler-Lagrange equation for (4) action

$$
\frac{d^{2} h}{d L^{2}} \frac{d L}{d q} \frac{\partial L}{\partial v}+\frac{d h}{d L} \frac{d}{d q} \frac{\partial L}{\partial v}+\frac{d}{d q} \frac{\partial L_{1}}{\partial v}=\frac{d h}{d L} \frac{\partial L}{\partial x}+\frac{\partial L_{1}}{\partial x},
$$


with the same for (3) action

$$
k \frac{d}{d q} \frac{\partial L}{\partial v}+\frac{d}{d q} \frac{\partial L_{1}}{\partial v}=k \frac{\partial L}{\partial x}+\frac{\partial L_{1}}{\partial x} .
$$

The corollary: the (1) action and the (2) action are equivalent. To see this, we need to set

$$
h(L)=-m c \sqrt{L}, \quad L=g_{a b} \frac{d x^{a}}{d q} \frac{d x^{b}}{d q}, \quad L_{1}=-\frac{e}{c} A_{a} \frac{d x^{a}}{d q}
$$

The first condition is satisfied by the same Euler's homogeneous function theorem, because the Hamiltonian for (2) action is proportional to the Lagrangian from (5), since $L$ is quadratic in velocities, and $L_{1}$ is linear in velocities. The second condition: $k$ from (3) is exactly equal to the derivative of $h(L): k=\frac{d h}{d L}=-\frac{m c}{2 \sqrt{L}}$. This coefficient stands before the first term in the (2) action. $I$ denotes a persistent value of $L$ from (5), that is the interval squared. Usually [1-4] a natural parameter $s$ is taken instead of arbitrary $q$. They are connected by a simple formula $d s=\sqrt{I} d q$, which follows from the comparison of $d s$ and $I$.

Let's write down the Euler-Lagrange equations for (1) and (2) actions. In contrast to the usual derivations [1-4], we assume the value of the interval is not a unity, but $\sqrt{I}$.

$$
\frac{m c}{\sqrt{I}} \frac{d}{d q}\left(g_{a b} \frac{d x^{b}}{d q}\right)+\frac{e}{c} \frac{d}{d q} A_{a}=\frac{m c}{2 \sqrt{I}} \frac{\partial g_{b c}}{\partial x^{a}} \frac{d x^{b}}{d q} \frac{d x^{c}}{d q}+\frac{e}{c} \frac{\partial A_{b}}{\partial x^{a}} \frac{d x^{b}}{d q}
$$

From the (6) system, it can be seen that without electromagnetic fields, $\frac{m c}{\sqrt{I}}$ factors are reduced, and the equations do not depend on which parameter is taken, the interval $s$ or the initial $q$ parameter. But if electromagnetic field is present, there will be different equations. A transition to the natural parameter $s$ is possible, which follows from (6). However, this possibility is absent in multiparticle problems, as we will see below.

\section{Multiparticle Problem, Time Synchronization, Hamiltonian Formulation and Liouville Equation}

Let's consider a multiparticle problem of motion in a gravitational and electromagnetic field. Consider an action similar to (1) for an ensemble of particles.

$$
S_{1}=-\sum_{r} m_{r} c \int \sqrt{g_{a b} \frac{d x_{r}^{a}}{d q} \frac{d x_{r}^{b}}{d q}} d q-\sum_{r} \frac{e_{r}}{c} \int A_{a} \frac{d x_{r}^{a}}{d q} d q
$$

We can again come to (2)-type Lagrangian and get an equivalent action

$$
S=-\sum_{r} \frac{m_{r} c}{2 \sqrt{I_{r}}} \int g_{a b}(x) \frac{d x_{r}^{a}}{d q} \frac{d x_{r}^{b}}{d q} d q-\sum_{r} \frac{e_{r}}{c} \int A_{a} \frac{d x_{r}^{a}}{d q} d q
$$


It should be noted that here an $r$ index appears which numbers the particles. Values of $I_{r}$ integral denoting the particles' interval sizes are not necessarily the same. Here we have synchronized the proper time of the particles $d s_{r}=\sqrt{I_{r}} d q$ in the following sense: 1) we have showed that the impossibility of synchronizing the $d s_{r}$ intervals themselves is come from the different values of $I_{r} ; 2$ ) we have showed how different proper times are related: the $q$ parameter for all particles is the same. It's important that the $I_{r}$ integrals depend on parameterization, but their ratio does not, so it's convenient to rewrite the action (8) via the $d s$ interval of some particle (observer).

$$
S=-\sum_{r} \frac{m_{r} c \sqrt{I}}{2 \sqrt{I_{r}}} \int g_{a b}(x) \frac{d x_{r}^{a}}{d s} \frac{d x_{r}^{b}}{d s} d s-\sum_{r} \frac{e_{r}}{c} \int A_{a} \frac{d x_{r}^{a}}{d s} d s .
$$

Using the usual momentum formulae, from (2) or (7) or (8) we get

$$
Q_{r a}=\frac{\partial L}{\partial v_{r}^{a}}=-\frac{m_{r} c}{\sqrt{I_{r}}} g_{a b}\left(x_{r}\right) \frac{d x_{r}^{b}}{d q}-\frac{e_{r}}{c} A_{a}\left(x_{r}\right)
$$

These $Q_{r a}$ are the canonical «long» momenta [1-4]. Then the velocities expressed through the long momenta are

$$
\begin{gathered}
\frac{d x_{r}^{b}}{d q}=-\frac{\sqrt{I_{r}}}{m_{r} c} g^{a b}\left(x_{r}\right)\left(Q_{r a}+\frac{e_{r}}{c} A_{a}\right) \\
\frac{d Q_{r a}}{d q}=\sum_{r} \frac{\sqrt{I_{r}}}{m_{r} c}\left(Q_{r d}+\frac{e_{r}}{c} A_{d}\left(x_{r}\right)\right) \frac{\partial g d b}{\partial x^{a}}\left(x_{r}\right)\left(Q_{r b}+\frac{e_{r}}{c} A_{b}\left(x_{r}\right)\right)+ \\
\frac{e_{r} \sqrt{I_{r}}}{m_{r} c^{2}}\left(Q_{r d}+\frac{e_{r}}{c} A_{d}\left(x_{r}\right)\right) g d b \frac{\partial A_{d}}{\partial x_{r}^{a}}
\end{gathered}
$$

There is a Hamiltonian for which the equations (10) and (11) are canonical:

$$
H=\sum_{r} \frac{\sqrt{I_{r}}}{m_{r} c}\left(Q_{r a}+\frac{e_{r}}{c} A_{a}\left(x_{r}\right)\right) g^{a b}\left(x_{r}\right)\left(Q_{r b}+\frac{e_{r}}{c} A_{b}\left(x_{r}\right)\right)
$$

The integrals $\sqrt{I_{r}}$ do the time synchronization here, leading to differentiation by the same parameter $q$. Now we write the corresponding Liouville equation for the distribution function $f_{r}(x, p, q)$.

$$
\begin{gathered}
\frac{\partial f_{r}}{\partial q}-\frac{\sqrt{I_{r}}}{m_{r} c} g^{a b}(x)\left(Q_{a}+\frac{e_{r}}{c} A_{a}\right) \frac{\partial f_{r}}{\partial x^{b}}+ \\
\left(\frac{\sqrt{I_{r}}}{m_{r} c}\left(Q_{d}+\frac{e_{r}}{c} A_{d}(x)\right) \frac{\partial g d b}{\partial x^{a}}\left(Q_{b}+\frac{e_{r}}{c} A_{b}(x)\right)+\right. \\
\left.\frac{e_{r} \sqrt{I_{r}}}{m_{r} c^{2}}\left(Q_{d}+\frac{e_{r}}{c} A_{d}(x)\right) g d b \frac{\partial A_{d}}{\partial x^{a}}\right) \frac{\partial f_{r}}{\partial Q_{a}}=0
\end{gathered}
$$

Here indices $r$ have moved from the momenta and the coordinates to the distribution function $f_{r}(x, p, q)$ as usual [5-13], and the equations depend on the indices only through mass, charges and intervals squared, i.e. $I_{r}$. 
Let's write out the stationary form of this equation, where $f_{r}(x, p, q)$ does not depend on $q$, this is how the Vlasov-Einstein equation is usually written $[5,10,11]$.

$$
\begin{gathered}
-g^{a b}(x)\left(Q_{r b}+\frac{e_{r}}{c} A_{b}\right) \frac{\partial f_{r}}{\partial x^{a}}+ \\
\left(\frac{\partial g^{b d}}{\partial x^{a}}\left(Q_{r d}+\frac{e_{r}}{c} A_{d}\right)\left(Q_{r b}+\frac{e_{r}}{c} A_{b}\right)+\right. \\
\left.\frac{e_{r}}{c} F_{a b}(x) g^{d b}\left(Q_{r d}+\frac{e_{r}}{c} A_{d}\right)\right) \frac{\partial f_{r}}{\partial p_{a}}=0
\end{gathered}
$$

The integrals $I_{r}$ and the masses $m_{r}$ have disappeared, but the electric charges $e_{r}$ have not. Compare now the resulting equations with those when using «short» momenta in (7) without the electromagnetic field.

$$
p_{r a}=-\frac{m_{r} c}{\sqrt{I_{r}}} g_{a b}\left(x_{r}\right) v_{r}^{b}, \quad v_{r}^{b}=\frac{d x_{r}^{b}}{d q}
$$

We have obtained the first-order equations for the (7) (or (8)). They are not Hamiltonian, but divergence-free. For the sake of clarity, we will use the proper time of some particle (observer) $s, d s=\sqrt{I} d q$ when synchronizing, instead of the affine parameter $q$.

$$
\begin{gathered}
\frac{d x_{r}^{b}}{d s}=-\frac{\sqrt{I_{r}}}{m_{r} c \sqrt{I}} g^{a b}\left(x_{r}\right) p_{r a}, \\
\frac{d}{d s}\left(p_{r a}\right)=-\frac{\sqrt{I_{r}}}{m_{r} c \sqrt{I}} \frac{\partial g^{b d}}{\partial x^{a}} p_{r b} p_{r d}+\frac{e_{r}}{c} \frac{\sqrt{I_{r}}}{m_{r} c \sqrt{I}} F_{a b}\left(x_{r}\right) g^{d b}\left(x_{r}\right) p_{r d}
\end{gathered}
$$

Let's write the Liouville equation for the distribution function $f_{r}(x, p, s)$ of particles with masses $m_{r}$ and electric charges $e_{r}$ over the 4-space $x$, 4-momentum $p$ with $s$ as a parameter.

$$
\begin{gathered}
\frac{\partial f_{r}}{\partial s}-\frac{\sqrt{I_{r}}}{m_{r} c \sqrt{I}} g^{a b}(x) p_{a} \frac{\partial f_{r}}{\partial x^{b}}+ \\
\left(-\frac{\sqrt{I_{r}}}{m_{r} c \sqrt{I}} \frac{\partial g^{b d}}{\partial x^{a}} p_{b} p_{d}+\frac{e_{r}}{c} \frac{\sqrt{I_{r}}}{m_{r} c \sqrt{I}} F_{a b}(x) g^{d b} p_{d}\right) \frac{\partial f_{r}}{\partial p_{a}}=0
\end{gathered}
$$

The stationary form of this equation, i.e. $f_{r}(x, p, s)$ does not depend on $s$ :

$$
-g^{a b}(x) p_{a} \frac{\partial f_{r}}{\partial x^{b}}+\left(-\frac{\partial g^{b d}}{\partial x^{a}} p_{b} p_{d}+\frac{e_{r}}{c} F_{a b}(x) g^{d b} p_{d}\right) \frac{\partial f_{r}}{\partial p_{a}}=0
$$

So, we have obtained the stationary ((13) or (17)) Liouville equations, also nonstationary ((12) or (16)) ones. We can see that when transforming (12) to (13) or (16) to (17) the integrals $\sqrt{I_{r}}$ and the masses $m_{r}$ are being reduced, but the charges $e_{r}$ are not. 


\section{Stationary Solution Analysis}

It can be seen from (6) that in the stationary case, when the metric $g_{a b}$ and vector potentials $A_{a}$ do not depend on the time coordinate $x_{0}=c t$, the right-hand side in (6) disappears when $a=0$, and we can integrate the left side

$$
\frac{m c}{\sqrt{I}}\left(g_{0 b} \frac{d x^{b}}{d q}\right)+\frac{e}{c} A_{0}=-Q_{0}
$$

\subsection{Landau Metric}

To understand what the integral (18) is, let's take the non-relativistic metric [3, Eq. (87.13)]:

$$
g_{a b}=\left(1+\frac{2 U}{c^{2}},-1,-1,-1\right)
$$

Then the integral (18) becomes

$$
\frac{m c}{\sqrt{I}}\left(1+\frac{2 U}{c^{2}}\right)\left(\frac{d x^{0}}{d q}\right)+\frac{e}{c} A_{0}=-Q_{0}
$$

The remaining equations (6) take the following form

$$
\frac{m c}{\sqrt{I}} \frac{d}{d q}\left(\frac{d x^{j}}{d q}\right)+\frac{e}{c} \frac{d}{d q} A_{j}=\frac{m c}{c^{2} \sqrt{I}} \frac{\partial U}{\partial x^{j}}\left(\frac{d x^{0}}{d q}\right)^{2}+\frac{e}{c} \frac{\partial A_{b}}{\partial x^{j}} \frac{d x^{b}}{d q}, \quad j=1,2,3 .
$$

We can exclude $q$ differentiation, replacing it with $x^{0}$ or $t$ differentiation, and the equations will acquire a familiar form of dynamics in the electromagnetic field with Lorentz force and electrostatics and gravitational potential $U$, but the effective mass expression is quite interesting.

$$
\frac{d}{d t}\left(M \frac{d x^{j}}{d t}\right)=-M \frac{\partial U}{\partial x^{j}}+\frac{e}{c} F_{b j} \frac{d x^{b}}{d t}
$$

Here $F_{a b}$ are common field expressions by potentials [1-13], and the expression for effective mass $M$ is

$$
M=-\frac{\frac{Q_{0}}{c}+\frac{e}{c^{2}} A_{0}}{1+\frac{2 U}{c^{2}}}
$$

We can see how the effective mass (22) depends on gravitational and electric fields, therefore $Q_{0}$ can be considered as zero component of the momentum or energy outside the fields. It should be noted that all calculations are accurate when using the Lagrangian (2). Let's write out the expression for $Q_{0}$, replacing $q$ differentiation with $t$ one in (18), also get the final expression for the effective mass.

$$
Q_{0}=-\frac{m c\left(1+\frac{2 U}{c^{2}}\right)}{\sqrt{1-\frac{v^{2}}{c^{2}}+\frac{2 U}{c^{2}}}}-\frac{e}{c} A_{0}, \quad M=\frac{m}{\sqrt{1-\frac{v^{2}}{c^{2}}+\frac{2 U}{c^{2}}}}
$$




\subsection{Fock Metric}

Let's consider now the Fock metric [2]

$$
g_{a b}=\left(1+\frac{2 U}{c^{2}},-\left(1-\frac{2 U}{c^{2}}\right),-\left(1-\frac{2 U}{c^{2}}\right),-\left(1-\frac{2 U}{c^{2}}\right)\right)
$$

The equation of motion takes a more complex form than (21)

$$
\frac{d}{d t}\left(M \frac{d x^{j}}{d t}\right)=-M \frac{1+\frac{v^{2}}{c^{2}}}{1-\frac{2 U}{c^{2}}} \frac{\partial U}{\partial x^{j}}+\frac{e}{c} F_{b j} \frac{d x^{b}}{d t}
$$

The $Q_{0}$ and $M$ expressions are as follows

$$
Q_{0}=-\frac{m c\left(1+\frac{2 U}{c^{2}}\right)}{\sqrt{1-\frac{v^{2}}{c^{2}}+\frac{2 U}{c^{2}}+\frac{2 U v^{2}}{c^{4}}}}-\frac{e}{c} A_{0}, \quad M=-\frac{\left(\frac{Q_{0}}{c}+\frac{e}{c^{2}} A_{0}\right)\left(1-\frac{2 U}{c^{2}}\right)}{1+\frac{2 U}{c^{2}}}
$$

Finally, for the effective mass we get

$$
M=\frac{m\left(1-\frac{2 U}{c^{2}}\right)}{\sqrt{1-\frac{v^{2}}{c^{2}}+\frac{2 U}{c^{2}}+\frac{2 U v^{2}}{c^{4}}}}
$$

\section{Uniform Universe: Solutions that Depend Only on Time}

Let the metric and the gravitational and electromagnetic fields depend only on time, which means that the universe in completely uniform. In this case, the (6) equations can be integrated via Hamiltonian mechanics, but it is interesting to look at specific aspects. We have three motion integrals

$$
\frac{m c}{\sqrt{I}}\left(g_{d b} \frac{d x^{b}}{d q}\right)+\frac{e}{c} A_{d}=Q_{d}, \quad d=1,2,3
$$

We use the integral of «energy» instead of the equation for the zero component, that is the interval squared.

$$
I=g_{a b} \frac{d x^{a}}{d q} \frac{d x^{b}}{d q}
$$

Now we get that all small momenta are determined as time functions from (14) and (23):

$$
p_{d}=\frac{e}{c} A_{d}-Q_{d}, \quad d=1,2,3
$$


The last zero component is determined as a time function from the analogue of the «energy» integral, that is the square of the interval for the momenta.

$$
g^{a b} p_{a} p_{b}=m^{2} c^{2}
$$

Here we come to a well-known relation that leads to the Hamilton-Jacobi method [1-12]. The equations for determining all coordinates are

$$
\frac{d x^{a}}{d q}=-\frac{\sqrt{I}}{m c} g d a\left(x^{0}\right) p_{d}
$$

We can exclude $q$ via dividing the three equations (23) for $d=1,2,3$ by the equation for $d=0$.

$$
\frac{d x^{a}}{d x^{0}}=\frac{g^{d a}\left(x^{0}\right) p_{d}\left(x^{0}\right)}{g^{0 d}\left(x^{0}\right) p_{d}\left(x^{0}\right)}=\frac{g^{d a}\left(x^{0}\right)\left(\frac{e}{c} A_{d}\left(x^{0}\right)-Q_{d}\right)}{g^{0 d}\left(x^{0}\right)\left(\frac{e}{c} A_{d}\left(x^{0}\right)-Q_{d}\right)}, \quad a=1,2,3
$$

We have obtained equations where terms depend only on time, and these equations can be easy integrated. The solutions are significant generalizations of de Sitter space [12]. Such equations would be appropriate to be applied to the question of dark energy and dark matter $[13,14]$.

\section{Vlasov-Maxwell-Einstein Equations}

When deriving the Vlasov-Maxwell-Einstein equations according to [6-9, 13], we use the classical action [1-5]:

$$
\begin{aligned}
S= & -\sum_{r, \lambda} m_{r} c \int \sqrt{g_{a b} \frac{d x_{r, \lambda}^{a}}{d q} \frac{d x_{r, \lambda}^{b}}{d q}} d q-\sum_{r, \lambda} \frac{e_{r}}{c} \int A_{a} \frac{d x_{r, \lambda}^{a}}{d q} d q \\
& -\frac{1}{16 \pi c} \int F_{a b} F^{a b} \sqrt{-g} d^{4} x+k \int(R+\Lambda) \sqrt{-g} d^{4} x
\end{aligned}
$$

Here $k=\frac{-c^{3}}{16 \pi \gamma}$, and $\Lambda$ is the cosmological constant [1-4]. The particles are divided into classes indexed by $r$ with different masses and electric charges, also individual particles are indexed by $\lambda$ inside each class.

In order to obtain field equations and relate the fields with the distribution function $f_{r}(x, p, q)$, we need to rewrite the first two terms of (25) via this distribution function, then do the variation by fields. Let's rewrite (28), replacing $q$ with $t$.

$$
\begin{aligned}
S= & -\sum_{r, \lambda} m_{r} c \int \sqrt{g_{a b} \frac{d x_{r, \lambda}^{a}}{d t} \frac{d x_{r, \lambda}^{b}}{d t}} d t-\sum_{r, \lambda} \frac{e_{r}}{c} \int A_{a} \frac{d x_{r, \lambda}^{a}}{d t} d t \\
& -\frac{1}{16 \pi c} \int F_{a b} F^{a b} \sqrt{-g} d^{4} x+k \int(R+\Lambda) \sqrt{-g} d^{4} x
\end{aligned}
$$


We can express the velocities through the momenta, excluding $q$ by dividing all the equations from (15) by the equation for zero component.

$$
\frac{d x^{a}}{d t}=c \frac{g^{d a}(x) p_{d}}{g^{0 d}(x) p_{d}}, \quad a=1,2,3,4
$$

Indices $r, \lambda$ are omitted here, and we took into account that $t=\frac{x^{0}}{c}$. Substituting (30) instead of velocities in (29), we get the action expressed through momenta.

$$
\begin{aligned}
S= & -\sum_{r, \lambda} m_{r} c^{2} \int \frac{\sqrt{p_{a}^{r \lambda} g d a\left(x_{r \lambda}\right) p_{d}^{r \lambda}}}{g^{d 0}\left(x_{r \lambda}\right) p_{d}^{r \lambda}} d t-\sum_{r, \lambda} e_{r} \int \frac{A_{a} g^{d a}\left(x_{r \lambda}\right) p_{d}^{r \lambda}}{g^{d 0}\left(x_{r \lambda}\right) p_{d}^{r \lambda}} d t \\
& -\frac{1}{16 \pi c} \int F_{a b} F^{a b} \sqrt{-g} d^{4} x+k \int(R+\Lambda) \sqrt{-g} d^{4} x
\end{aligned}
$$

Next, we replace summation over $\lambda$ by integration over momenta and space with distribution function $f_{r}(x, p), x \in R^{4}, p \in R^{4}$ :

$$
\begin{aligned}
S= & -\sum_{r} m_{r} c^{2} \int \frac{\sqrt{p_{a} g^{d a}(x) p_{d}}}{g^{d 0}(x) p_{d}} f_{r}(x, p) d^{4} x d^{4} p \\
& -\sum_{r} e_{r} \int \frac{A_{a} g^{d a}(x) p_{d}}{g^{d 0}(x) p_{d}} f_{r}(x, p) d^{4} x d^{4} p \\
& -\frac{1}{16 \pi c} \int F_{a b} F^{a b} \sqrt{-g} d^{4} x+k \int(R+\Lambda) \sqrt{-g} d^{4} x
\end{aligned}
$$

It is impossible to find out experimentally which terms in (32) we deal with. At present, experiments show that the Lambda depends on time. This fact can be instantly obtained from (32), as well as dependence on space.

Reverse transition from the action (32) to the action (31) can be done by substitution $f_{r}(x, p)=\sum_{\lambda} \delta\left(x-x_{r \lambda}(t)\right) \delta\left(p-p_{r \lambda}(t)\right)$, which can be considered as a verification. So, we got the derivation scheme of the Vlasov-Maxwell-Einstein equations. Let's consider the expression (32), taking into account that the cosmological constant is now used for dark energy modeling $[14,15]$. The first three terms of $(28)$ action can play the role of $\Lambda$-term in (32), therefore the dark energy can be composed of these three terms with some coefficients.

$$
\begin{aligned}
\Lambda_{D E}(x)= & -\frac{\lambda_{f}}{16 \pi c k} F_{a b} F^{a b}-\lambda_{p} \sum_{r} \frac{m_{r} c^{2}}{\sqrt{-g} k} \int \frac{\sqrt{p_{a} g^{d a}(x) p_{d}}}{g^{d 0}(x) p_{d}} f_{r}(x, p) d^{4} p \\
& -\lambda_{p f} \sum_{r} \frac{e_{r}}{\sqrt{-g} k} \int \frac{A_{a} g^{d a}(x) p_{d}}{g^{d 0}(x) p_{d}} f_{r}(x, p) d^{4} p
\end{aligned}
$$




$$
\begin{aligned}
\Lambda_{M}(x)= & -\frac{\left(1-\lambda_{f}\right)}{16 \pi c k} F_{a b} F^{a b}-\left(1-\lambda_{p}\right) \sum_{r} \frac{m_{r} c^{2}}{\sqrt{-g} k} \int \frac{\sqrt{p_{a} g^{d a}(x) p_{d}}}{g^{d 0}(x) p_{d}} f_{r}(x, p) d^{4} p \\
& -\left(1-\lambda_{p f}\right) \sum_{r} \frac{e_{r}}{\sqrt{-g} k} \int \frac{A_{a} g^{d a}(x) p_{d}}{g^{d 0}(x) p_{d}} f_{r}(x, p) d^{4} p
\end{aligned}
$$

Here DE (Dark Energy) and M (Matter) stand for dark energy and matter, respectively. One can take into account the contributions of any other fields in the same way. In fact we got the opportunity not to introduce Einstein's $\Lambda$ a priori, but to get its counterpart from the way how classical Lagrangians influence on matter. The signs of electrostatic energy and $\Lambda$ match here, so, apparently, the repulsion of dark energy is an electrostatic repulsion, and most of the matter which is seen as dark energy, coincides with the cosmic plasma. There is no need to consider any other candidates for dark energy, since we know that there are only two types of long-range actions (gravity and electromagnetism), and any others would have revealed themselves already. This can be well seen from the non-relativistic counterparts of the action, that will be described in the other place. The trace of dark energy was found too: its mathematical contribution to the action is the same as that of Einstein's $\Lambda$.

The Vlasov-Einstein-Maxwell equations for the metric and the electric fields are obtained by varying the action (32) by them. First we will vary by metric and get

$$
\begin{aligned}
k\left(R_{a b}-\frac{1}{2} R-\frac{\Lambda}{2}\right) \sqrt{-g}= & \sum_{r} m_{r} c^{2} \int\left(\frac{1}{2 p^{0} \sqrt{p^{d} p_{d}}}-\frac{\sqrt{p^{d} p_{d}}}{\left(p^{0}\right)^{2} p_{0}} \delta_{0}^{b}\right) f_{r}(x, p) p_{a} p_{b} d^{4} p+ \\
& \sum_{r} e_{r} \int\left(\frac{\left(A_{a} p_{b}+A_{b} p_{a}\right)}{2 p^{0}}-\frac{A_{d} p^{d}}{\left(p^{0}\right)^{2} p_{0}} p_{a} p_{b} \delta_{0}^{b}\right) f_{r}(x, p) d^{4} p \\
& +\frac{1}{16 \pi c} F_{d c} F^{d c}\left(-\frac{1}{2} \sqrt{-g}\right) g_{a b}
\end{aligned}
$$

Now we will vary the electromagnetic potentials. We obtain the Maxwell equation in the gravitational field.

$$
\frac{2}{16 \pi c} \frac{\partial\left(\sqrt{-g} F^{a b}\right)}{\partial x^{b}}=\sum_{r} e_{r} \int \frac{g^{d a}(x) p_{d}}{g^{d 0}(x) p_{d}} f_{r}(x, p) d^{4} p
$$

Finally, we got the Vlasov-Maxwell-Einstein equation system (17), (33), (34).

\section{Conclusion}

So, we have derived the Vlasov-Maxwell-Einstein equation. There was necessary to synchronize the proper times of different particles. We did this in two ways, first 
via the proper time of a single particle, second via an arbitrary parameter. Such parameters in various sources are called differently: sometimes affine [12, 35], sometimes canonical [36].

We have derived equations and obtained expressions for effective mass in stationary gravitational and electromagnetic fields for the two metrics. Conversely, we have got solutions that depend only on time. It's interesting to compare the obtained form of the Vlasov-Maxwell-Einstein equations with other versions and to classify them. Usually they are written out only for the Vlasov-Einstein equations and with Christoffel symbols, and therefore not for momenta but for velocities [10, 11, 16, 17]. They can also be derived according to our scheme. When these equations are not derived, but written immediately as given ones, inaccuracies may occur. When it comes to Vlasov-Einstein equations, the deriving seems necessary for the both Liouville equation and field equation. When deriving the Liouville equation, the time synchronization arise. The energy-momentum tensor in the field equations has to be taken arbitrarily, if there is no deriving.

In the transition from (31) to (32) we have obtained the expressions which formally have the same effect as the Einstein's lambda. It seems promising to research all classical substitutions for this equation that are well-known for the Vlasov equation: energy and hydrodynamic ones [6-9]. It's also interesting to investigate the stationary solutions [18-25]. The problem of classifying all time-dependent (spatially homogeneous) solutions is relevant and interesting too, because it leads to cosmological solutions, which are now being actively studied. The Hamilton-Jacobi equation methods [26-31] would be useful here. A very important task is to obtain for the Vlasov-like equations a statement like «Time averages coincide with the Boltzmann extremals» [32-34]. The authors thank N.N. Fimin, V.M. Chechetkin, A.D. Chernin, K.A. Bronnikov, S.O. Alekseev for useful discussions. 


\section{References}

[1] W. Pauli. Theory of relativity. Courier Corporation, 1981.

[2] V. Fock. The theory of space, time and gravitation. Elsevier, 2015.

[3] L. D. Landau. The classical theory of fields. Vol. 2. Elsevier, 2013.

[4] B. A. Dubrovin, A. T. Fomenko, and S. P. Novikov. Modern geometry-methods and applications: Part II: The geometry and topology of manifolds. Vol. 104. Springer Science \& Business Media, 2012.

[5] A. Vlasov. Statistical distribution functions. Nauka, Moscow, 1966.

[6] V. V. Vedenyapin and M. Negmatov. "Derivation and classification of Vlasovtype and magnetohydrodynamics equations: Lagrange identity and Godunov's form”. In: Theoretical and Mathematical Physics 170.3 (2012), pp. 394-405.

[7] V. V. Vedenyapin, M. A. Negmatov, and N. N. Fimin. "Vlasov-type and Liouvilletype equations, their microscopic, energetic and hydrodynamical consequences". In: Izvestiya: Mathematics 81.3 (2017), p. 505.

[8] V. V. Vedenyapin and M.-B. A. Negmatov. "On derivation and classification of Vlasov type equations and equations of magnetohydrodynamics. The Lagrange identity, the Godunov form, and critical mass". In: Contemporary Mathematics. Fundamental Directions 47 (2013), pp. 5-17.

[9] A. Sinitsyn, V. Vedenyapin, and E. Dulov. Kinetic Boltzmann, Vlasov and related equations. Elsevier, 2011.

[10] Y. Choquet-Bruhat. General relativity and the Einstein equations. Oxford University Press, 2009.

[11] G. Kremer and C. Cercignani. The Relativistic Boltzmann Equation: Theory and Applications. 2002.

[12] J. V. Narlikar. An introduction to cosmology. Cambridge University Press, 1993.

[13] V. Vedenyapin. Boltzmann and Vlasov kinetic equations. 2001.

[14] A. D. Chernin. "Dark energy and universal antigravitation". In: Physics-Uspekhi 51.3 (2008), pp. 253-282.

[15] K. F. Valiyev and A. Kraiko. "The dispersion of an ideal gas from a point into a void. A new model of the Big Bang and the expansion of the Universe". In: Journal of Applied Mathematics and Mechanics 79.6 (2015), pp. 556-565.

[16] G. Rein and A. D. Rendall. "Global existence of solutions of the spherically symmetric Vlasov-Einstein system with small initial data". In: Communications in mathematical physics 150.3 (1992), pp. 561-583. 
[17] Y. G. Ignatyev. "The Nonequilibrium Universe: The Kinetics Models of the Cosmological Evolution". In: (2014).

[18] V. Vedeniapin. "A boundary value problem for stationary Vlasov equations". In: Akademiia Nauk SSSR Doklady 290.4 (1986), pp. 777-780.

[19] Y. Y. Arkhipov and V. V. Vedenyapin. "On the classification and stability of steady-state solutions of Vlasov's equation on a torus and in a boundary value problem". In: Trudy Matematicheskogo Instituta imeni VA Steklova 203 (1994), pp. 13-20.

[20] V. V. Kozlov. "The generalized Vlasov kinetic equation". In: Russian Mathematical Surveys 63.4 (2008), p. 691.

[21] V. V. Kozlov. "The Vlasov kinetic equation, dynamics of continuum and turbulence". In: Nelineinaya Dinamika [Russian Journal of Nonlinear Dynamics] 6.3 (2010), pp. 489-512.

[22] A. L. Skubachevskii and Y. Tsuzuki. "Vlasov-Poisson equations for a twocomponent plasma in a half-space". In: Doklady Mathematics 94.3 (2016), pp. 681-683.

[23] A. L. Skubachevskii. "Vlasov-Poisson equations for a two-component plasma in a homogeneous magnetic field". In: Russian Mathematical Surveys 69.2 (2014), p. 291.

[24] Y. O. Belyaeva. "Stationary solutions of Vlasov equations for high-temperature two-component plasma". In: Contemporary Mathematics. Fundamental Directions 62 (2016), pp. 19-31.

[25] J. Batt et al. "Some families of solutions of the Vlasov-Poisson system". In: Archive for Rational Mechanics and Analysis 104.1 (1988), pp. 79-103.

[26] V. Kozlov. "General vortex theory”. In: Dynamical Systems X. Springer, 2003, pp. 76-107.

[27] V. Vedenyapin and M. Negmatov. "On the topology of steady-state solutions of hydrodynamic and vortex consequences of the Vlasov equation and the Hamilton-Jacobi method". In: Doklady Mathematics. Vol. 87. 2. Springer. 2013, pp. 240-244.

[28] V. Vedenyapin and N. Fimin. "The Liouville equation, the hydrodynamic substitution, and the Hamilton-Jacobi equation". In: Doklady Mathematics. Vol. 86. 2. Springer. 2012, pp. 697-699.

[29] V. V. Vedenyapin, N. N. Fimin, and M. A. Negmatov. Vlasov and Liouvilletype equations and its microscopic and hydrodynamic consequences. 2016.

[30] V. V. Vedenyapin and N. N. Fimin. "The Hamilton-Jacobi method for nonHamiltonian systems”. In: Nonlinear Dynamics 11.2 (2015), pp. 279-286. 
[31] V. Vedenyapin and N. Fimin. "The Hamilton-Jacobi method in the non-Hamiltonian situation and the hydrodynamic substitution". In: Doklady Mathematics. Vol.91. 2. Springer. 2015, pp. 154-157.

[32] V. V. Vedenyapin and S. Z. Adzhiev. "Entropy in the sense of Boltzmann and Poincaré”. In: Russian Mathematical Surveys 69.6 (2014), p. 995.

[33] S. Z. Adzhiev and V. V. Vedenyapin. "Time averages and Boltzmann extremals for Markov chains, discrete Liouville equations, and the Kac circular model". In: Computational Mathematics and Mathematical Physics 51.11 (2011), pp. 1942 1952.

[34] V. Vedenyapin. "Time averages and Boltzmann extremals". In: Doklady Mathematics. Vol. 78. 2. Springer. 2008, pp. 686-688.

[35] A. P. Lightman et al. Problem book in relativity and gravitation. Princeton University Press, 2017.

[36] J. Synge and J. Romain. "Relativity: The general theory". In: Physics Today 14 (1961), p. 50.

[37] S. Z. Adzhiev et al. "Generalized Boltzmann-type equations for aggregation in gases". In: Computational Mathematics and Mathematical Physics 57.12 (2017).

[38] V. V. Vedenyapin, S. Z. Adzhiev, and V. V. Kazantseva. "Entropy in the sense of Boltzmann and Poincare, Boltzmann extremals, and the Hamilton-Jacobi method in non-Hamiltonian context". In: Contemporary Mathematics. Fundamental Directions 64.1 (2018), pp. 37-59.

[39] S. Adzhiev, V. Vedenyapin, and S. Filippov. "H-Theorem for Continuous-and Discrete-Time Chemical Kinetic Systems and a System of Nucleosynthesis Equations". In: Computational Mathematics and Mathematical Physics 58.9 (2018), pp. 1462-1476.

[40] V. V. Vedenyapin. "Vlasov-Maxwell-Einstein Equation”. In: Preprints of the Keldysh Institute of Applied Mathematics (2018).

[41] V. V. Vedenyapin, N. N. Fimin, and V. M. Chechetkin. "On the Vlasov-MaxwellEinstein equation and its non-relativistic and weakly relativistic analogues". In: Preprints of the Keldysh Institute of Applied Mathematics (2018), pp. 26530.

[42] A. V. Serghienko and V. A. Rubakov. "Phantom dark energy with tachyonic instability: metric perturbations". In: Theoretical and Mathematical Physics 173.3 (2012), pp. 1709-1719.

[43] V. N. Lukash and V. A. Rubakov. "Dark energy: myths and reality". In: PhysicsUspekhi 51.3 (2008), p. 283. 
Vedenyapin Victor Valentinovich, vicveden@yahoo.com

Keldysh Institute of Applied Mathematics (Russian Academy of Sciences), 125047, Moscow, Miusskaya square, 4

Peoples' Friendship University of Russia, 117198, Moscow, Miklouho-Maclay street, 6

Pershin Ilya Sergeevich, pershin@ phystech.edu

Keldysh Institute of Applied Mathematics (Russian Academy of Sciences), 125047, Moscow, Miusskaya square, 4

Moscow Institute of Physics and Technology, 141701, Moscow Region, Dolgoprudny, Institutskiy per., 9 


\section{Contents}

1 The Equivalence of Two Actions in the GTR and in the Theory of Geodesics ........................4 4

2 Multiparticle Problem, Time Synchronization, Hamiltonian Formulation and Liouville Equation . . . . . . . . . . . . . . . . . 5

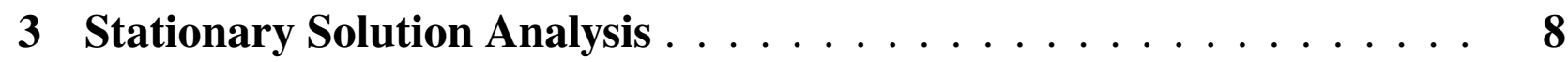

3.1 Landau Metric . . . . . . . . . . . . . . . . . . 8

3.2 Fock Metric . . . . . . . . . . . . . . . . . . . 9

4 Uniform Universe: Solutions that Depend Only on Time . . . . . . 9

5 Vlasov-Maxwell-Einstein Equations $\ldots \ldots \ldots \ldots$

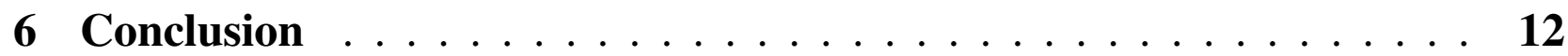

